\title{
Effects of yoga on health-related quality, physical health and psychological health in women with breast cancer receiving chemotherapy: a systematic review and meta-analysis
}

\author{
Li-Juan $\mathrm{Yi}^{1 \#}$, Xu Tian ${ }^{2,3 \#}$, Yan-Fei Jin ${ }^{2}$, Ming-Jie Luo ${ }^{4,5}$, Maria F. Jiménez-Herrera $^{2}$ \\ ${ }^{1}$ Department of Nursing, Hunan Traditional Chinese Medical College, Zhuzhou, China; ${ }^{2}$ Nursing Department, Universitat Rovira i Virgili, \\ Tarragona, Spain; ${ }^{3}$ Department of Gastroenterology, Chongqing University Cancer Hospital, School of Medicine, Chongqing University, \\ Chongqing, China; ${ }^{4}$ Xiangya School of Nursing, Central South University, Changsha, China; ${ }^{5}$ School of Nursing, Xinjiang Medical University, \\ Urumqi, China \\ Contributions: (I) Conception and design: LJ Yi, X Tian, MJ Luo, MF Jiménez-Herrera; (II) Administrative support: X Tian, MF Jiménez-Herrera; (III) \\ Provision of study materials or patients: LJ Yi, X Tian; (IV) Collection and assembly of data: LJ Yi, X Tian; (V) Data analysis and interpretation: LJ \\ Yi, X Tian; (VI) Manuscript writing: All authors; (VII) Final approval of manuscript: All authors. \\ \#These authors contributed equally to this work as co-first authors. \\ Correspondence to: Dr. Maria F. Jiménez-Herrera, PhD, MB, RN. Nursing Department, Universitat Rovira i Virgili, Avinguda Catalunya, 3543002 \\ Tarragona, Spain. Email: maria.jimenez@urv.cat.
}

Background Yoga receive more attention from breast cancer patients, however its feasibility and efficacy during chemotherapy remains conflicting. We performed this systematic review to assess the effects of yoga on health-related quality, physical health and psychological health in breast cancer patients undergoing chemotherapy.

Methods: A systematic search was conducted to retrieve randomized controlled trials (RCTs) which investigated the comparative efficacy of yoga versus comparators such as usual care among breast cancer patients for health-related quality, physical health and psychological health in PubMed, EMBASE, Cochrane Central Register of Controlled Trials (CNETRAL), Nursing and Allied Health Literature (CINAHL), Chinese Biomedical Literature (CBM) Database, China Science and Technology Journal (CSTJ) Database, China National Knowledge Infrastructure (CNKI), and Wangfang Database from inception to December 2018. The latest search was updated on September 2020. All analyses were completed using RevMan version 5.3.

Results: Seven trials involving 693 breast cancer patients met inclusion criteria. Meta-analysis indicated a short-term improvement in fatigue [standard mean difference (SMD), -0.62; 95\% confidence interval (CI), -1.17 to -0.07 ], sleep disturbance (SMD, $-0.34 ; 95 \%$ CI, -0.55 to -0.12 ), depression (SMD, $-0.50 ; 95 \%$ CI, -0.70 to -0.31 ) anxiety (SMD, $-0.50 ; 95 \% \mathrm{CI},-0.70$ to -0.31$)$, and health-related quality of life (QoL) (SMD, 0.72 ; $95 \% \mathrm{CI},-0.12$ to 1.56 ) in the yoga group; however beneficial medium- and long-term effects in fatigue, sleep disturbance were not identified. Moreover, qualitative analyses suggested that yoga was not associated with decreased adverse events (AEs) compared with control groups.

Conclusions: Yoga may benefit to reduce fatigue, depression and anxiety, improve sleep disturbance, and improve QoL in breast cancer patients receiving chemotherapy in the short-term; however, medium- and long-term effects should be further established owing to limitations.

Keywords: Brest cancer; chemotherapy; yoga; systematic review; meta-analysis

Submitted Jul 26, 2020. Accepted for publication Nov 03, 2020.

doi: 10.21037/apm-20-1484

View this article at: http://dx.doi.org/10.21037/apm-20-1484 


\section{Introduction}

Breast cancer represents a major public health problem of great concern (1), with approximately 2.09 million new cases and 0.63 million deaths in 2018 (2). As one of the comprehensive therapeutic strategies for breast cancer, chemotherapy is widely applied to breast cancer patient in respective less of stages (3). However, chemotherapy is often accompanied by numerous adverse effects such as fatigue, sleep disturbance, depression, and anxiety and eventually decrease quality of life (QoL), all of which may outweigh treatment benefits (4-9). Pharmacological interventions have already been confirmed to counter some of these adverse effects but potential drug toxicity is a concern $(10,11)$. Physical activity may be an attractive option for managing symptoms related to disease and treatment, because it can maintain psychological well-being and physical function, reduce cancer-related fatigue, mitigate breast cancer recurrence, and increase survival rates (12-15). In addition, physical activity can provide rehabilitative effects in QoL (16-18). Nevertheless, breast cancer survivors, especially who are receiving chemotherapy, often face severe physical fatigue and discomfort, and perceived barriers to participation in purely gymnastic exercise (19-22).

Compared to other exercise programs, yoga is highly flexible and adaptable and therefore has been used in cancer patients. Particularly, the postures of yoga can be easily modified to fit various functional impairments or limited mobility with harmless $(23,24)$. Therefore, yoga may act as a more viable or suitable alternative for breast cancer patients receiving chemotherapy. As a complementary therapy, yoga deems patients' spirituality as relevant for disease management (25). Evidence suggests that yoga can enhance body awareness and normalize the stress and immune response, consequently assist patients returning to optimal homeostasis (26-31). Several randomized clinical trials (RCTs) have been examined the role of yoga in breast cancer patients undergoing chemotherapy, most of which (32-37) established the benefits of yoga in relieving fatigue, sleep disturbance, depression, anxiety or improving health-related QoL. However, a study conducted by Chaoul et al. (38) reported a conflicting conclusion, in which total sleep disturbances or fatigue levels over time among three groups were not significantly different. Thus, a definitive conclusion on the efficacy and safety of yoga among breast cancer survivors who were prescribed to receive chemotherapy has not yet been obtained.

A recent meta-analysis (39) explored the role of yoga in breast cancer patients, and revealed an improvement in QoL and mental health. However, patients receiving radiotherapy and chemotherapy were not separately investigated in this study, and thus the pure effect of yoga on patients underwent chemotherapy alone could not be identified. Considering above issues, the primary objective of this systematic review is to further determine the potential benefits of yoga interventions for health-related quality, physical health and psychological health in breast cancer patients undergoing chemotherapy alone through pooling the results from eligible RCTs.

We present the following article in accordance with the PRISMA reporting checklist (available at http://dx.doi. org/10.21037/apm-20-1484).

\section{Methods}

This systematic review was carried out according to the recommendations released by the Cochrane Collaboration (40). We reported all results following the Preferred Reporting Items for Systematic Reviews and Meta-Analyses statement (PRISMA) statement (41) (details in Supplemental Digital Content 1). In this article, all analyses were performed based on published data, and thus the ethical approval and informed consent was not required. Moreover, the current systematic review and meta-analysis has not formal registered protocol.

\section{Selection criteria}

For inclusion, a trial should meet the following criteria: (I) patients $(P)$ : exclusively concerned women patients who were currently undergoing chemotherapy for breast cancer, regardless of dose and type of chemotherapy; (II) intervention $(I)$ : any types of yoga were viewed as the experimental intervention. Even if studies simply described the intervention as 'yoga' also met the requirement; (III) control $(C)$ : wait-list control, usual care, no therapy and any other active therapy were eligible as control intervention; (IV) outcomes (O): Health-related QoL, physical health (e.g., fatigue, sleep disturbance, nausea and vomiting) and psychological health (e.g., depression, anxiety and perceived stress) were regarded as the primary outcome. And if available, adherence and safety-related data served as secondary outcome measures; (V) study design (S): only RCTs published in English and Chinese language were considered. Full-text or abstract publications were accessed only when they can provide essential information on 
research design, characteristics of participants, interventions and outcomes.

Studies were excluded if it covered at least one of the following criteria: (I) yoga was not the main intervention but a part of a multi-modal intervention (e.g., MindfulnessBased Stress Reduction, mindfulness-based cognitive therapy), as the effects of yoga could not be isolated in such programs; (II) study was short of the essential information or primary data was unavailable to be obtained from authors; and (III) duplicates (develop from the same research project) with poor methodology and insufficient data would not be chosen.

\section{Definition of outcomes}

In this study, the QoL was assessed by using any validated generic or disease-specific self-report scale in each individual study. In terms of indicators related to physical or psychological health, we measured them by any validated self-report or clinician-rated scale in all individual trials. Safety-related data was acquired by monitoring and recording the number of women with adverse events (AEs).

\section{Identification of citations}

The following electronic databases were independently searched by two investigators including PubMed, EMBASE, Cochrane Central Register of Controlled Trials (CNETRAL), Nursing and Allied Health Literature (CINAHL), Chinese Biomedical Literature (CBM) database, Chinese Scientific and Technological Periodical (VIP) and China National Knowledge Infrastructure (CNKI), and Wangfang Database. All initial searches were completed by December 2018, with the latest search was updated on September 2020. The search terms including Breast Neoplasms, chemotherapy, Yoga and random were used to develop search strategy. The PubMed was used as an example to show how these search strategies were employed (details in Supplemental Digital Content 2). Besides, we scrutinized the reference lists of retrieved articles and relevant reviews manually to obtain any potential studies. After screening the titles and abstracts identified by the initial search, two independent investigators conducted full-text check to definitively determine eligibility. Any disagreements were resolved through discussion and consensus. We used the Kappa statistic to present the level of agreement between investigators.

\section{Data extraction and quality assessment}

A data extraction sheet was developed to extract essential information from the selected articles. We assigned two investigators to extract the following data: first author, publication year and country, age, sample size, stage of breast cancer, possible treatments, details of intervention regimes, and outcome measures. When multiple publications were identified from the same clinical trial, we cited the primary complete report of that trial. But data from all publications were extracted. It would introduce bias, if the same data were included repeatedly. Corresponding authors were contacted if the information of interest could not be extracted. Discrepancies were solved through discussion, if necessary, by consulting an independent a third reviewer.

We appraised the quality of individual studies using the Cochrane Risk of Bias Tool (42), which is based on the following six domains (40): randomization sequence generation, allocation concealment, blinding of participants, blinding of study personnel, blinding of outcome assessors, incomplete outcome data, selective reporting and other bias. Items were graded in high, low or unclear risk of bias (40). Any conflicting results were resolved by consensus and arbitration by a third party arbitrated.

\section{Statistical analysis}

All data were statistically analyzed by RevMan version 5.3 (Copenhagen, Denmark: The Nordic Cochrane Centre, The Cochrane Collaboration). Risk ratio (RR) with $95 \%$ confidence interval (CI) was used to express the dichotomous outcomes, while we mean differences (MDs) with 95\% CIs were calculated to express continuous outcomes. We described the heterogeneity in results across studies based on the Cochrane's Q statistic, and inconsistency was quantitatively estimate with the $\mathrm{I}^{2}$ statistic $(43,44)$ when clinical heterogeneity across eligible studies was not detected. When non-significant heterogeneity was detected $\left(\mathrm{I}^{2}<50 \%\right)$ across studies and a fixed-effects model was utilized to combine the data. In contrast, a randomeffects model was utilized. We drew a funnel plot to inspect the publication bias when more than 10 eligible studies reported a same outcome. We evaluated the robustness of our overall analyses by conducting subgroup analyses. For multiple-arm studies, outcome data were extracted from all groups which satisfy the inclusion criteria as recommended in the Cochrane Handbook for Systematic Reviews of 


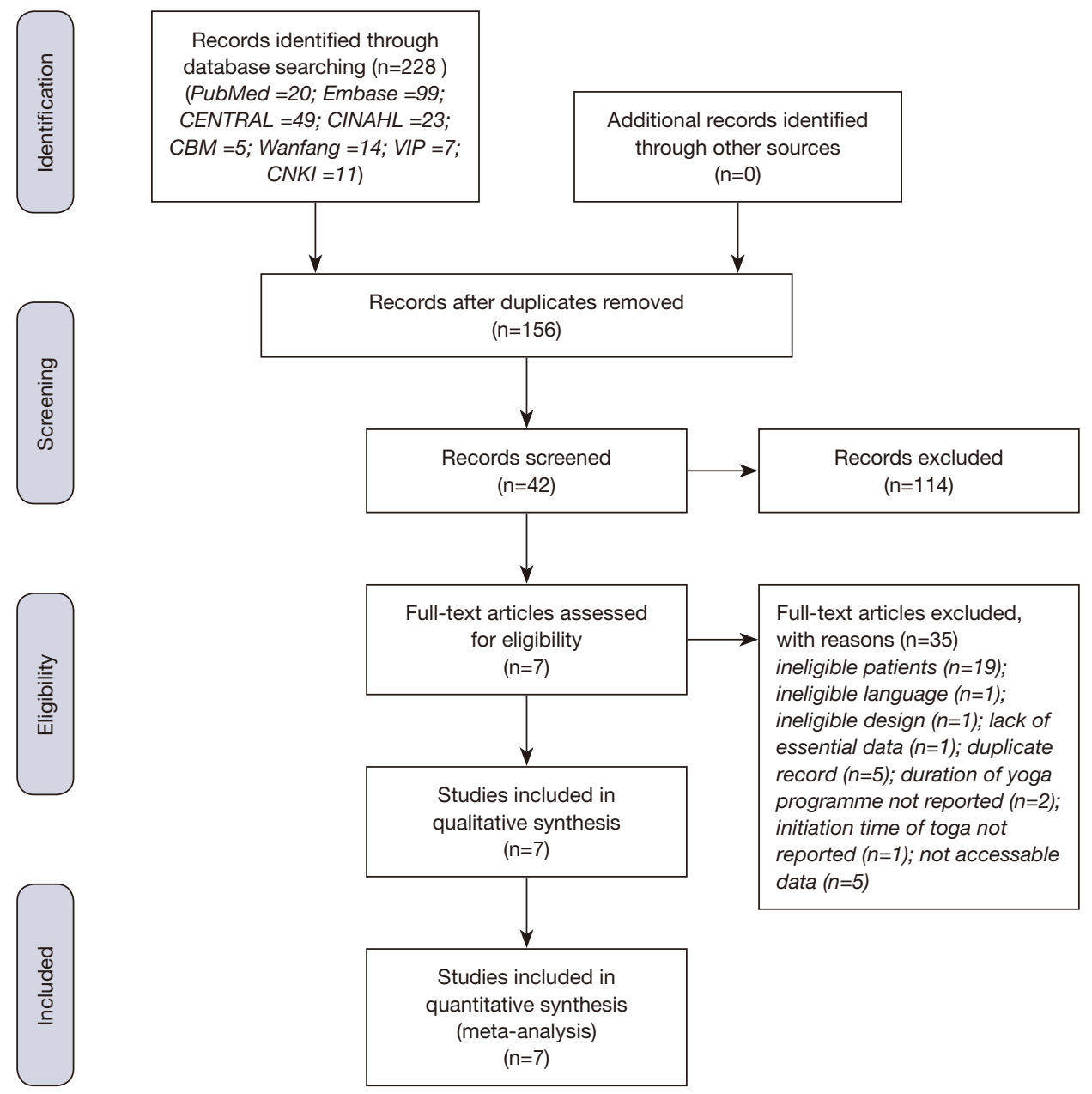

Figure 1 PRISMA flow diagram of retrieval and selection of literature. CNETRAL, Cochrane Central Register of Controlled Trials; CINAHL, Nursing and Allied Health Literature; CBM, Chinese Biomedical Literature; CSTJ, China Science and Technology Journal; CNKI, China National Knowledge Infrastructure; RCT, randomized controlled trial.

Interventions (40) and were reported individually. If the trials reported their continuous variables as mean with ranges, we would convert those variable into the mean and variance (standard deviation, SD) according to the method proposed by Hozo et al. (45). All disagreements between the investigators were discussed and resolved by consensus.

\section{Results}

\section{Identification and selection of studies}

Through a database search, a total of 228 potentially relevant records were retrieved, and no additional citation was added. After screening the title, abstract and full-text of 156 non-duplicate records, ultimately 7 articles published between 2013 and 2018 met our criteria and were included in qualitative synthesis. Of the 7 included studies, one (38) involving two control groups (yoga vs. usual care; yoga $v s$. exercise) was divided into two comparisons according to the purpose of the study. Besides, a small $(n=40)$ study conducted by Danhauer et al. (32) posted addition results in Clinical Trials.gov which didn't present in current paper, so we integrated all results. A flow chart outlining the process of literature identification and selection was depicted in Figure 1.

\section{Characteristics of eligible studies}

The basic characteristics of 7 trials ( 8 RCTs) were summarized in Table 1 . They were originated from China 


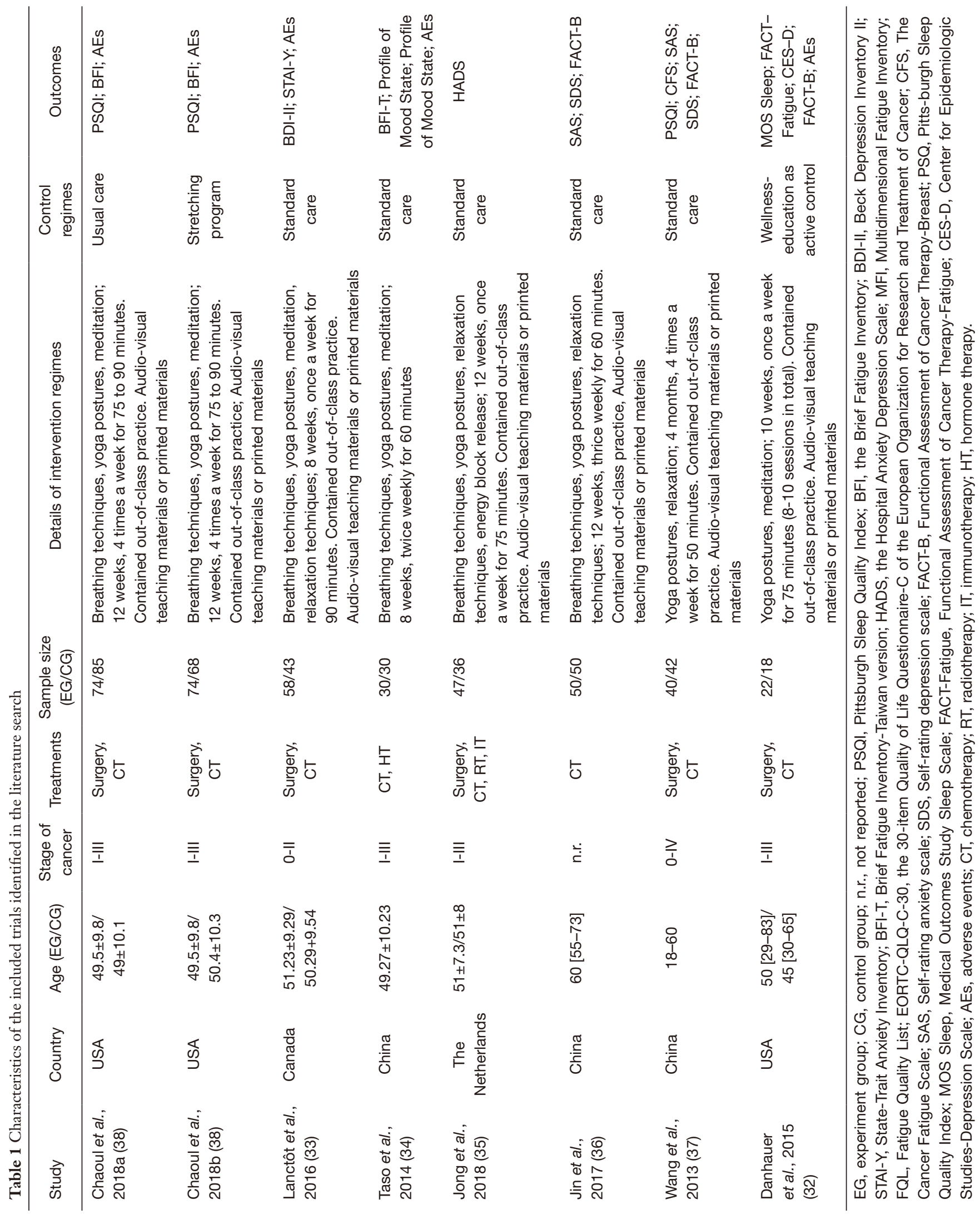


(3 trials), America (2 trials), Canada (1 trials), and The Netherlands (1 trials). The included studies recruited a total of 693 breast cancer patients, with sample size ranging from 40 to 159. Different yoga interventions were applied across the studies, including Tibetan, Bali, Anusara, Dru, and integrated yoga programs. The remaining two did not specify the yoga style adopted. The main instruction form is group coaching (5 trials) $(32-34,36,37)$ and participants in 3 studies (32-34) received personalized yoga sessions led by experienced yoga instructors. The yoga interventions varied in duration from 8 weeks to 4 mouths and in frequency from 1 to 4 times a week for 50 - to 90 -minute sessions. Besides, the time point of longest follow-up ranged from 0 to 12 months. As repeated outcome assessments were conducted, statistical analysis was undertook separately according to the duration of follow-up: short-term ( $<6$ months), medium-term (6-12 months) and long-term follow-up ( $\leq 12$ months). In 6 trials (32-37) were categorize as assessing short-term effects; the remaining one (38) as assessing short-, medium- and long-term effects.

\section{Methodological quality of studies}

All eligible articles were described as randomized, however, only five $(32-35,38)$ specified methods to generate random sequencing, such as a form of adaptive randomization or a computer-generated random table. Less than half of articles (33-35) provided sufficient information on allocation concealment, one study (37) fail to conceal allocation; and the remaining studies $(32,36,38)$ were no mention of methods used to do so. The control group in most studies consisted of either wait-list or usual care, considering participants were aware of which intervention they received, it was hard to imagine how blinding of patients and personnel could be applicable. Because a subjective judgment of participants and researchers played an important role in the evaluation of outcome indicators, and this phenomenon may have caused an obvious Hawthorne effects. Thus, we rated these studies (33-38) as having a high risk of bias. However, the remaining studies $(32,38)$ have used an active intervention as a control group whose frequency and duration is similar to yoga group, and no group differences was detected in target outcomes over time; thus it is unlikely to introduce bias without performing blinding (39). Five articles (34-38) reported no information on blinding of outcome assessors; and two $(32,33)$ explicitly indicated that the researchers responsible for the outcome assessment were not blinded. In aspect of attrition bias, no attrition $(34,36)$ and low attrition $(32,33,37)$ were detected in 2 and 3 studies, respectively. One study (38) reported high dropout rate in each group, but well balanced across groups. Besides, group differences were not statistically significant between study completers versus non-completers on the basis of demographic and medical characteristics and outcome measures; and the remaining one (35) was graded as high risk of bias because a dropout rate $>20 \%$ ) (46). All studies provided no protocol but there was no hint of selective reporting, except for one study (35) that registered protocol and reported all pre-specified outcomes in a prespecified way. No study suffered from other suggested bias, and we judged all studies as having a low risk of bias in this domain. We displayed a summary of the quality assessments in Figure 2.

\section{Health-related QoL}

With respect to health-related QoL, three RCTs $(32,36,37)$ involving 216 participants investigated its short-term effects of yoga. No heterogeneity was detected across the included studies $\left(\mathrm{P}=0.24, \mathrm{I}^{2}=29 \%\right)$, and then a random-effect model was adopted to calculate estimate. Pooled result suggested that the efficacy of yoga interventions is not inferior to the control groups in health-related QoL [SMD $=0.72,95 \%$ CI, -0.12 to $1.56, \mathrm{P}=0.09$ ] (Figure 3).

\section{Fatigue}

Four trials $(32,34,37,38)$ comprising 442 patients investigated whether yoga can relieve cancer-related fatigue in short-term period. We divided the eligible studies into three subgroups based on different assessment period. Heterogeneity was low among two studies that fell into medium-term effects group $\left(\mathrm{P}=0.85, \mathrm{I}^{2}=0 \%\right)$ and long-term effects group $\left(\mathrm{P}=0.89, \mathrm{I}^{2}=0 \%\right)$, but statistical heterogeneity presented among five RCTs that fell into short-term effects group $\left(\mathrm{P}<0.000, \mathrm{I}^{2}=86 \%\right)$. Therefore, a random-effects model was used. Meta-analysis revealed that statistically significant effects was detected on this dimension immediately postintervention [SMD $=-0.62,95 \% \mathrm{CI},-1.17$ to $-0.07, \mathrm{P}=0.03$ for short-term effects group]. However, the efficacy of yoga interventions returned to baseline at followup assessment $[\mathrm{SMD}=0.22,95 \% \mathrm{CI},-0.05$ to $0.49, \mathrm{P}=0.85$ for medium-term effects group and $\mathrm{SMD}=0.16,95 \% \mathrm{CI}$, -0.13 to $0.44, \mathrm{P}=0.89$ for long-term effects group] (Figure 4). 


\section{Sleep disturbance}

Regarding sleep disturbance, three trials $(32,37,38)(348$ participants) assessed short-term effects of yoga group versus control group. Among them, one RCTs (32) cannot provide available data to be used in meta-analysis. Thereby, we adopted a qualitative analysis. We divided the eligible studies into three subgroups based on different evaluation period. The heterogeneity test indicated

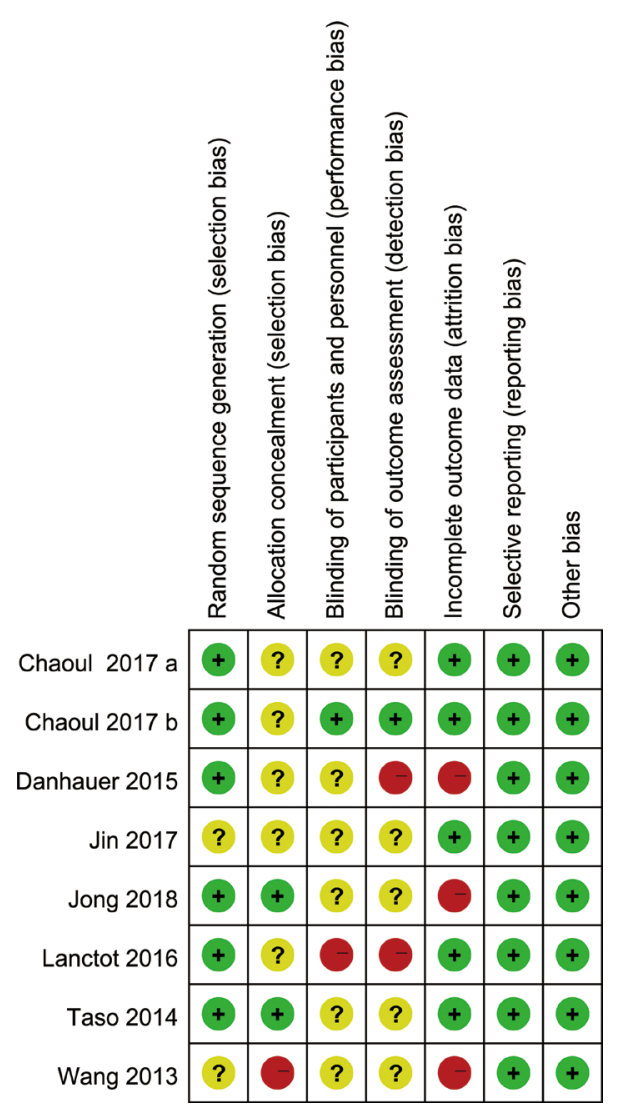

Figure 2 Risk of bias summary. Green, yellow and red represents low, unclear and high risk of bias respectively. statistical heterogeneity exists in short-term effects group $\left(\mathrm{P}=0.09, \mathrm{I}^{2}=59 \%\right.$ for short-term effects group) and no heterogeneity exists in remaining subgroups $(\mathrm{P}=0.50$, $\mathrm{I}^{2}=0 \%$ for medium-term effects group and $\mathrm{P}=0.73, \mathrm{I}^{2}=0 \%$ for long-term effects group). Therefore, a random-effects model was used. Pooled result suggested that yoga groups were significantly more effective than control groups in improving sleep disturbance when short-term effects was assessed (SMD $=-0.34,95 \% \mathrm{CI},-0.55$ to $-0.12, \mathrm{P}=0.003$ ). However, the efficacy of yoga interventions was unable to sustain at follow-up assessment $(\mathrm{SMD}=-0.09,95 \% \mathrm{CI}$, -0.36 to $0.18, \mathrm{P}=0.53$ for medium-term group and $\mathrm{SMD}$ $=0.00,95 \% \mathrm{CI},-0.28$ to $0.29, \mathrm{P}=0.98$ for long-term group) (Figure 5). Besides, Danhauer et al. (32) found that sleep disturbance from baseline to chemotherapy weeks 5 and 10 remained essentially unchanged for yoga participants $(\mathrm{P}>0.05)$.

\section{Depression}

A total of six RCTs (32-37) compared short-term effects of yoga in 446 women on this outcome. Considerable heterogeneity was detected across the included studies $\left(\mathrm{P}<0.000, \mathrm{I}^{2}=84 \%\right)$. Therefore, a random-effects model was used. Pooled result suggested that yoga groups were effective in improving this negative emotion as compared to control groups (SMD $=-0.56,95 \% \mathrm{CI},-1.05$ to -0.07 , $\mathrm{P}=0.03$ ) (Figure 6).

\section{Anxiety}

Five RCTs (33-37) that enrolled 412 participants reported short-term effects of yoga on fatigue. Study results were homogeneous $\left(\mathrm{P}=0.11, \mathrm{I}^{2}=46 \%\right)$. Therefore, a fixed-effects model was used. The pooled SMD was -0.50 (95\% CI, -0.70 to $-0.31, \mathrm{P}<0.000)$, indicating statistically significant benefit from yoga (Figure 7).

\begin{tabular}{|c|c|c|c|c|c|c|c|c|c|c|c|c|}
\hline \multirow[b]{2}{*}{ Study or Subgroup } & \multicolumn{3}{|c|}{ Yoga } & \multicolumn{3}{|c|}{ Control } & \multirow{2}{*}{\multicolumn{2}{|c|}{$\begin{array}{l}\text { Mean Difference } \\
\text { IV, Random, } 95 \% \mathrm{CI}\end{array}$}} & \multirow{2}{*}{\multicolumn{4}{|c|}{$\begin{array}{c}\text { Mean Difference } \\
\text { IV, Random, } 95 \% \mathrm{Cl}\end{array}$}} \\
\hline & Mean & SD & Total & Mean & SD & Total & & & & & & \\
\hline Danhauer 2015 & 103.89 & 20.33 & 18 & 102.89 & 20.33 & 16 & $5.4 \%$ & $1.00[-12.69,14.69]$ & & & & \\
\hline Jin 2017 & 96.34 & 8.38 & 50 & 83.15 & 10.62 & 50 & $44.7 \%$ & $13.19[9.44,16.94]$ & & & & \\
\hline Wang 2013 & 94.13 & 7.16 & 40 & 81.72 & 8.54 & 42 & $49.9 \%$ & $12.41[9.01,15.81]$ & & & & \\
\hline Total $(95 \% \mathrm{Cl})$ & & & 108 & & & 108 & $100.0 \%$ & $12.14[8.88,15.40]$ & & & & \\
\hline $\begin{array}{l}\text { Heterogeneity: } \mathrm{Tau}^{2}= \\
\text { Test for overall effect: }\end{array}$ & $\begin{array}{l}2.53 ; \mathrm{Chi}^{2} \\
Z=7.30(\end{array}$ & $\begin{array}{l}2=2.83 \\
(P<0.0\end{array}$ & $\begin{array}{l}\mathrm{df}=2 \\
0001)\end{array}$ & $(P=0.24$ & $4) ; 1^{2}=2$ & $29 \%$ & & & -20 & $-10 \underset{\text { yoga }}{0} 0$ & $\begin{array}{l}0 \quad 10 \\
\text { control }\end{array}$ & 20 \\
\hline
\end{tabular}

Figure 3 Meta-analysis of health-related quality of life. 


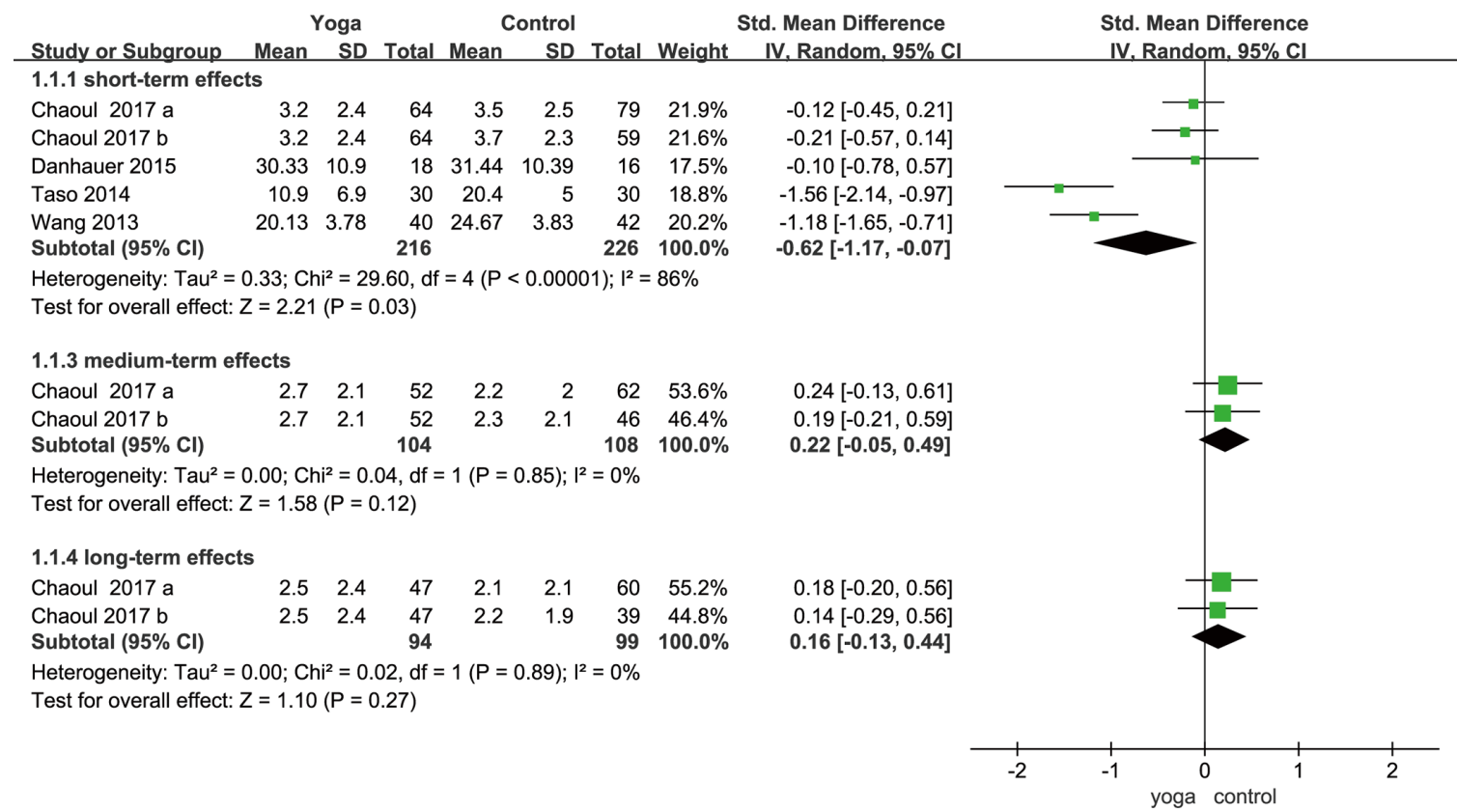

Figure 4 Meta-analysis of fatigue.

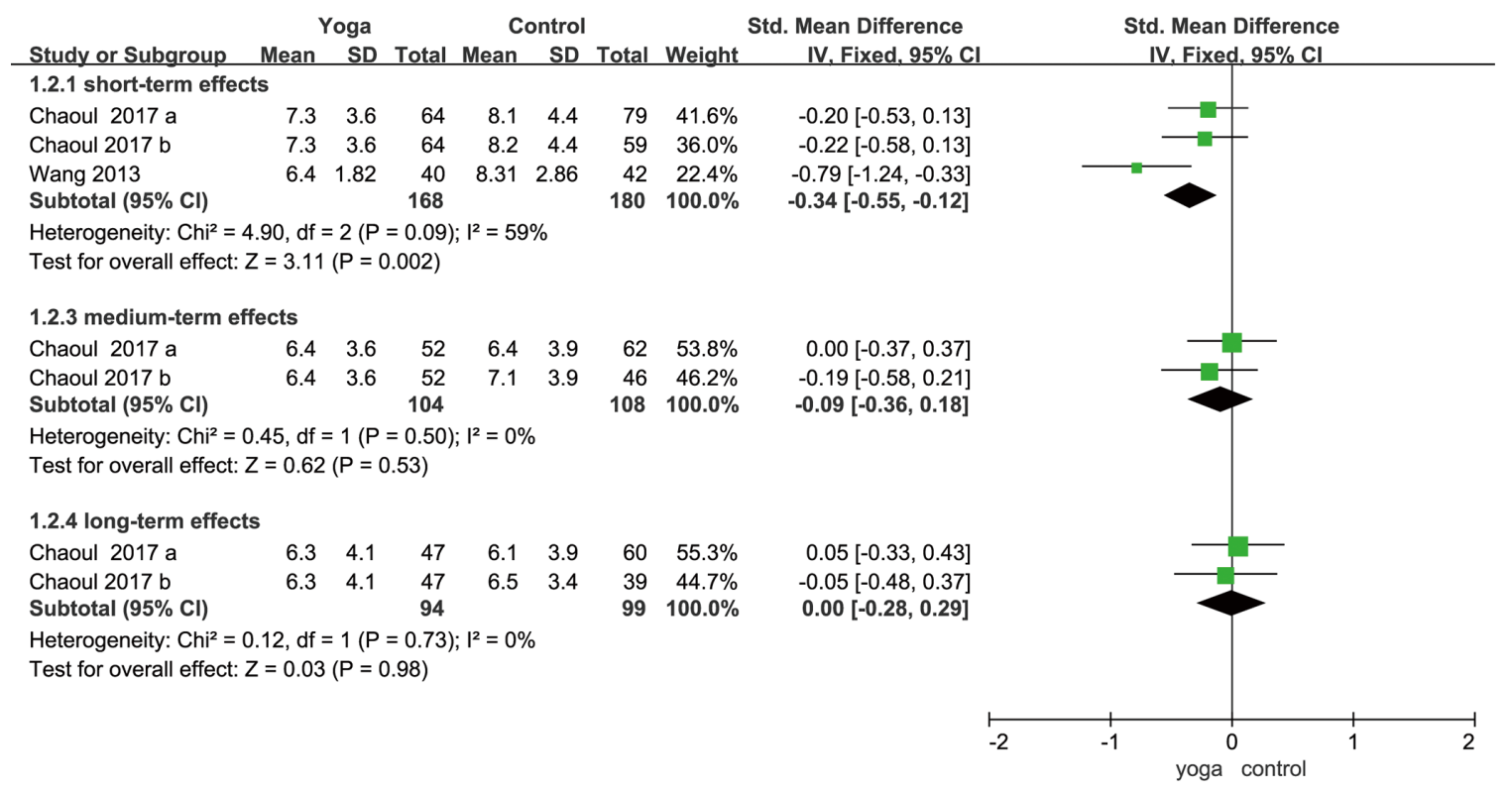

Figure 5 Meta-analysis of sleep disturbance.

\section{Safety}

Across the 7 included studies, in roughly half studies (32-34,38) provided the safety-related data. However, those numerical data cannot be used in meta-analysis. Thus, we took a descriptive analysis to summarize all information.

The first one (32) was conducted by Danhauer et al. elaborated on this outcome: of the 21 participants in the yoga group, $4.76 \%$ (1 patient) experienced serious $\mathrm{AE}$ and $52.38 \%$ (11 patients) experienced minor AEs; of the 17 participants in the educational wellness group, $5.88 \%$ (1 patient) experienced serious AE, 47.06\% (8 patients) 


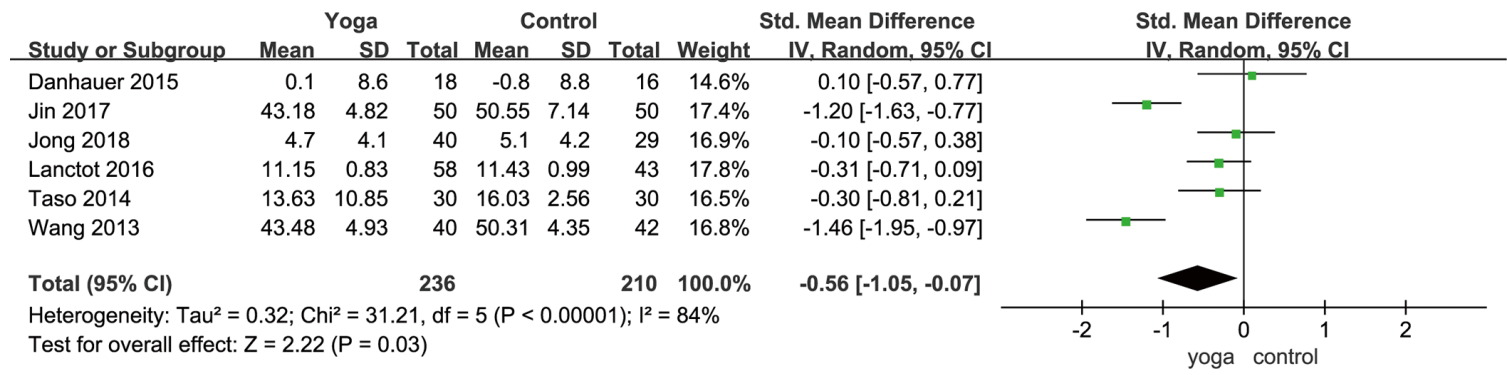

Figure 6 Meta-analysis of depression.

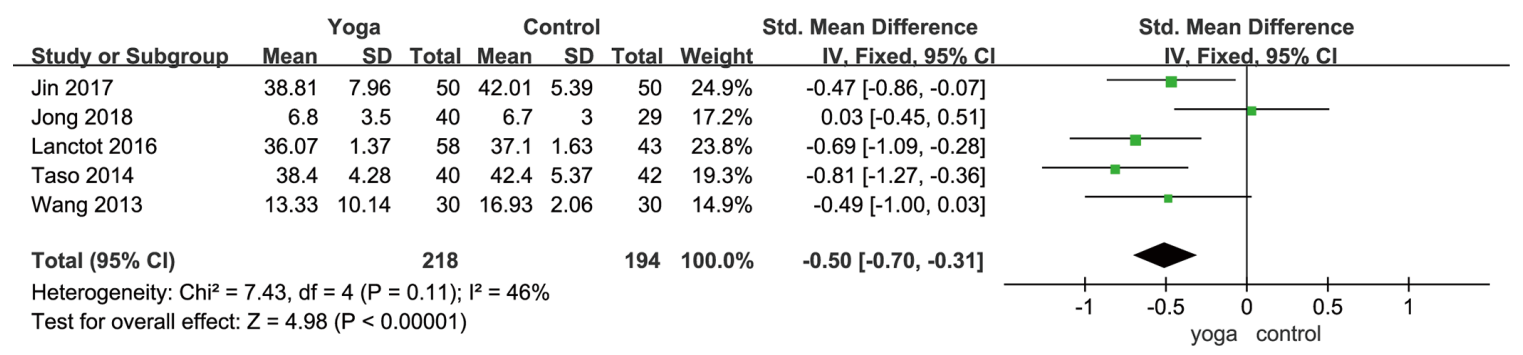

Figure 7 Meta-analysis of anxiety.

experienced minor AEs. The second trial (34) showed that a total of 57 AEs occurred by $81 \%$ (25 out of 31 ) women in the yoga group and 45 AEs occurred by $80 \%$ (20 out of 25) women in the control groups. The authors also clearly stated that none of the AEs was caused by yoga practice. Moreover, the remaining studies $(33,38)$ found no remarkable side effects was collected in both groups.

\section{Publication bias}

In this review, the small number of studies $(\mathrm{n}<10)$ were included in our quantitative analysis, and thus we decided not to perform a publication bias test based on a funnel plot.

\section{Discussion}

Yoga is an ancient Indian philosophy and science which emphasizes the mind, body and spirit flowing in perfect harmony with nature, and considers practitioners' spirituality as an essential addition for disease management (25,47-49). It seems to yield positive effect in diverse medical conditions such as diabetes $(50,51)$, asthma $(52,53)$, AIDS (54), heart failure $(55,56)$, immune function (57), serum cortisol levels (58) and Lymphoma (59). Previous researches (59-61) have also demonstrated that participants practicing yoga regularly have a substantial reduction in their natural killer cells and less DNA damage while undergoing chemotherapy (62).

Breast cancer, a leading cause of death in women between 35 and 54 years of age (2), is often treated with chemotherapy to reduce risk of distant recurrence (63). In this process, nevertheless, a myriad of undesired side effects ranging from physical (e.g., pain, fatigue, dry mouth, insomnia, nausea, vomiting) to psychological (e.g., emotional distress, anxiety, depression) $(7,9,64,65)$, all of which may impact substantially on QoL and activities of daily living, challenged breast cancer survivors to cope with. A trial conducted in breast cancer participants who are receiving chemotherapy found that $46 \%$ of them reported self-perceived cognitive impairments and $82 \%$ of them were subjected to cancer-related fatigue in at least one dimension (66). It is now widely acknowledged that exercise can significantly maintain physical fitness and function and mitigate sleep dysfunction and mood disturbance for women with breast cancer during chemotherapy (12-14,67). However, cancer-related pain and severe fatigue condition discouraged women with breast cancer from taking part in purely gymnastic exercise $(19,68)$. In comparison to other forms of physical activity, yoga's slow, gentle movements are easy to be adjusted to individual's medical conditions and the degree of activity limitation on the premise of guaranteeing 
safe $(20,69)$. Despite few published studies exclusively recruited breast cancer survivors receiving chemotherapy, information on the safety and efficacy of yoga practices is limited and inconsistent. Moreover, those results haven't been recur. In light of these findings and yoga's increasing popularity in this target population, to further confirm whether yoga is beneficial becomes paramount.

\section{Summary of main results}

To our knowledge, this is the first systematic review and meta-analysis to assess the strength of the evidence for yoga as a positive intervention for women with breast cancer receiving chemotherapy alone. A majority of studies only focused on short-term effects. The result indicated that yoga has an immediate effect on relieving fatigue and sleep disturbances, attenuating depression and anxiety and enhancing overall health-related QoL during chemotherapy compared to the control group. According to Cohen's categories (70), all comparisons indicated either a medium or a large effect size for yoga group over control conditions which suggested group differences might be clinically relevant. Nonetheless, most of the analyses had considerable heterogeneity which could not be reduced by subsequent subgroup analyses. So this likely affected the interpretation of the effect size. Besides, a total of 2 RCTs from an identical study investigated medium-term and longterm effects of yoga on sleep disturbance and fatigue, which stated no statistically significant effects. Given the lack of evidences from more researches and small sample, the results on the basis of these data pieces must be cautiously interpreted. Furthermore, we found no evidence of effects favoring yoga group over control group for increasing the incidence of minor or serious side effects.

Based on the published evidences, the mechanisms proposed to explain how yoga can exert short-term physical and psychological benefits involved the following aspects: (I) yoga has the capability of altering activity of the hypothalamic-pituitary-adrenal axis in response to stress $(26,71)$, thus decreasing the secretion of cortisol and suppressing the stress-induced physiological arousal (72-74). Ultimately, it may lead to the decrease in the anxiety and depression levels (74,75); (II) under stressful conditions, yoga can increase parasympathetic dominance involving deepen breath amplitude and reduced heart rate and blood pressure-all of which lighten the mood (76). (III) Yoga stimulates the human body to lower levels of cytokines [e.g., interleukin-6 (77), lymphocyte-1B (78)] to prevent the impairment of cellular immunity under high-stress situation (79-84). Several studies have found that changes in levels of interleukin-6 (IL-6) are significantly correlated with changes in fatigue $(13,79,80)$; (IV) through the progression of a series of static physical postures, yoga adopted stretching resulting in the increased muscular strength and endurance, which may have achieve invigorating effects on mental and physical energy when facing stress (85), and thereby may reduce fatigue and sleep disturbance $(34,86,87)$. When the severity of one of these symptoms is moderate or above, they often co-occur $(88,89)$. And these changes often have directly or (and) indirectly impact on health-related QoL $(90,91)$

However, the present study failed to find medium- and long-term effects of yoga on fatigue and sleep disturbance during chemotherapy. But for all this, it is still worthwhile to explore possible reasons contributing to the findings. The effectiveness of yoga may be associated to the treatment status (during active cancer treatment $v s$. completion of active treatment) (92), a previous meta-analysis revealed evidence of efficacy only for yoga when participants are undergoing active cancer treatment but not when they completed active treatment (93). Secondly, the severity level of fatigue and sleep disturbance were low when assessed at 6 and 12 months. Therefore, there was not much room for improvement, which may be a reason for the non-significant findings (94). Thirdly, the frequency of practice may be also an influence factor of treatment outcome (86). If the frequency of training was too poor, it is hard to produce observable effects (95). The only one included article (38) reported that breast cancer patients who practiced yoga $\geq 2$ times per week during their chemotherapy treatment were less likely to experience sleep problems at 6 months.

\section{Limitations of the present study}

Several limitations in our study warrant comment. The safety of yoga practice undergoing chemotherapy has been questioned. Although several randomized trials $(32-35,38)$ found no evidence of increased risk of AEs associated with yoga for women with breast cancer under chemotherapy. But reporting standards have not been unified yet, some studies not only minored intervention-related side effects, but also recorded follow-up toxicity data (32) or chemotherapy-associated data $(32,34,35)$. These evidences can't specially embody the safety of yoga. Besides, as only one trial (38) included a follow-up assessment 12 months post intervention, medium- and long-term effects could 
not be assessed for all pre-specified outcome indicators and quantitative analyses were unable to implement. Thus, it is unclear how sustainable the benefit of yoga would be beyond the active intervention period. Furthermore, there still remain some variability in yoga interventions (i.e., yoga styles and their components; the length and intensity of yoga), patient groups (i.e., stage of cancer; accepted or accepting treatments) and instruments used to assess the outcome. This may be reflected by the high heterogeneity $\left(\mathrm{I}^{2}>59 \%\right)$ among some comparisons. Because the trails included were randomized, there is a high probability that they would have been evenly distributed across all intervention arms. These limitations have hindered researchers from determining the true benefits of a yoga regimen to treat all outcomes of breast cancer.

\section{Implications for practice and further research}

In our review, only two RCTs $(32,38)$ have included an active control group, relying instead on nonactive control conditions such as a waiting list or usual care condition. While class frequency or duration in the control intervention cannot be tailored and matched to that in the yoga intervention, I wonder whether this factor influences intervention efficacy since chemotherapy. A previous studies done by Pan et al. (96) revealed that any such yoga effects are probably larger among individuals with breast cancer who had a yoga practice over longer periods of time. But American Cancer Society guidelines recommended that participants using chemotherapy and (or) radiotherapy who already take exercise may need to lower the intensity and duration of exercise sessions during treatment (97). Thereby, after confirming optimum practice duration criterion, future studies should examine whether a similarly time-intensive physically oriented approach (e.g., stretching) is efficacious when applied to breast cancer survivors under chemotherapy.

\section{Conclusions}

Based on limited data, yoga can be deemed as a supportive intervention for improving short term fatigue, sleep disturbance, depression, anxiety and health-related QoL in women with a diagnosis of breast cancer who are currently undergoing chemotherapy. But yoga may not produce any medium- and long-term benefits in reduction of sleep disturbance and fatigue. Given that evidence showing the safety of yoga is promising but preliminary, further studies are warranted to closely minoring and reporting intervention-related safety information.

\section{Acknowledgments}

We would like to express our warm appreciation to those who published all eligible studies included in the current systematic review and meta-analysis. Moreover, all authors also appreciate Ms. Hui Chen because, as his wife, she fully supports Dr. Xu Tian to dedicate most efforts and their precious time to this work.

Funding: None.

\section{Footnote}

Reporting Checklist: The authors have completed the PRISMA reporting checklist. Available at http://dx.doi. org/10.21037/apm-20-1484

Conflicts of Interest: All authors have completed the ICMJE uniform disclosure form (available at http://dx.doi. org/10.21037/apm-20-1484). The authors have no conflicts of interest to declare.

Ethical Statement: The authors are accountable for all aspects of the work in ensuring that questions related to the accuracy or integrity of any part of the work are appropriately investigated and resolved.

Open Access Statement: This is an Open Access article distributed in accordance with the Creative Commons Attribution-NonCommercial-NoDerivs 4.0 International License (CC BY-NC-ND 4.0), which permits the noncommercial replication and distribution of the article with the strict proviso that no changes or edits are made and the original work is properly cited (including links to both the formal publication through the relevant DOI and the license). See: https://creativecommons.org/licenses/by-nc-nd/4.0/.

\section{References}

1. World Cancer Research Fund (WCRF). Breast cancer statistics: Breast cancer is the most common cancer in women worldwide. 2018. Available online: https://www. wcrf.org/dietandcancer/cancer-trends/breast-cancerstatistics

2. Bray F, Ferlay J, Soerjomataram I, et al. Global cancer statistics 2018: GLOBOCAN estimates of incidence and 
mortality worldwide for 36 cancers in 185 countries. CA Cancer J Clin 2018;68:394-424.

3. Sakurada T, Bando S, Zamami Y, et al. Prophylactic administration of granulocyte colony-stimulating factor in epirubicin and cyclophosphamide chemotherapy for Japanese breast cancer patients: a retrospective study. Cancer Chemother Pharmacol 2019;84:1107-14.

4. Silberholz J, Bertsimas D, Vahdat L. Clinical benefit, toxicity and cost of metastatic breast cancer therapies: systematic review and meta-analysis. Breast Cancer Res Treat 2019;176:535-43.

5. Alarid-Escudero F, Blaes AH, Kuntz KM. Trade-offs Between Efficacy and Cardiac Toxicity of Adjuvant Chemotherapy in Early-Stage Breast Cancer Patients: Do Competing Risks Matter? Breast J 2017;23:401-9.

6. Huang HP, Wen FH, Yang TY, et al. The effect of a 12week home-based walking program on reducing fatigue in women with breast cancer undergoing chemotherapy: A randomized controlled study. Int J Nurs Stud 2019;99:103376.

7. Demiralp M, Oflaz F, Komurcu S. Effects of relaxation training on sleep quality and fatigue in patients with breast cancer undergoing adjuvant chemotherapy. J Clin Nurs 2010;19:1073-83.

8. American Cancer Society. Chemotherapy for breast cancer. [cited 2018 5/1]. 2017. Available online: https://www. cancer.org/cancer/breast-cancer/treatment/chemotherapyfor-breast-cancer.html

9. Lobo SA, Fernandes AFC, Almeida PC, et al. Quality of life in women with breast cancer undergoing chemotherapy. Acta Paul Enferm 2014;27:554-9.

10. Spring LM, Zangardi ML, Moy B, et al. Clinical Management of Potential Toxicities and Drug Interactions Related to Cyclin-Dependent Kinase 4/6 Inhibitors in Breast Cancer: Practical Considerations and Recommendations. Oncologist 2017;22:1039-48.

11. Chan VT, Yeo W. Antiemetic therapy options for chemotherapy-induced nausea and vomiting in breast cancer patients. Breast Cancer (Dove Med Press) 2011;3:151-60.

12. Yang CY, Tsai JC, Huang YC, et al. Effects of a homebased walking program on perceived symptom and mood status in postoperative breast cancer women receiving adjuvant chemotherapy. J Adv Nurs 2011;67:158-68.

13. van Waart H, Buffart LM, Stuiver MM, et al. Adherence to and satisfaction with low-intensity physical activity and supervised moderate-high intensity exercise during chemotherapy for breast cancer. Support Care Cancer
2020;28:2115-26.

14. Courneya KS, Segal RJ, Mackey JR, et al. Effects of exercise dose and type on sleep quality in breast cancer patients receiving chemotherapy: a multicenter randomized trial. Breast Cancer Res Treat 2014;144:361-9.

15. Nock NL, Owusu C, Kullman EL, et al. A CommunityBased Exercise and Support Group Program in AfricanAmerican Breast Cancer Survivors (ABCs). J Phys Ther Health Promot 2013;1:15-24.

16. Wengström Y, Bolam KA, Mijwel S, et al. Optitrain: a randomised controlled exercise trial for women with breast cancer undergoing chemotherapy. BMC Cancer 2017;17:100.

17. Fairman CM, Focht BC, Lucas AR, et al. Effects of exercise interventions during different treatments in breast cancer. J Community Support Oncol 2016;14:200-9.

18. Dong X, Yi X, Gao D, et al. The effects of the combined exercise intervention based on internet and social media software (CEIBISMS) on quality of life, muscle strength and cardiorespiratory capacity in Chinese postoperative breast cancer patients:a randomized controlled trial. Health Qual Life Outcomes 2019;17:109.

19. Courneya KS, McKenzie DC, Reid RD, et al. Barriers to supervised exercise training in a randomized controlled trial of breast cancer patients receiving chemotherapy. Ann Behav Med 2008;35:116-22.

20. El-Hashimi D, Gorey KM. Yoga-Specific Enhancement of Quality of Life Among Women With Breast Cancer: Systematic Review and Exploratory Meta-Analysis of Randomized Controlled Trials. J Evid Based Integr Med 2019;24:2515690x19828325.

21. Sharma M, Haider T, Knowlden AP. Yoga as an alternative and complementary treatment for cancer: a systematic review. J Altern Complement Med 2013;19:870-5.

22. Wang G, Wang S, Jiang P, et al. Effect of Yoga on cancer related fatigue in breast cancer patients with chemotherapy. Zhong Nan Da Xue Xue Bao Yi Xue Ban 2014;39:1077-82.

23. Kiecolt-Glaser JK, Bennett JM, Andridge R, et al. Yoga's impact on inflammation, mood, and fatigue in breast cancer survivors: a randomized controlled trial. J Clin Oncol 2014;32:1040-9.

24. Koula MJ, Knight JM. Increasing provider awareness of and recommendations for yoga and meditation classes for cancer patients. Support Care Cancer 2018;26:3635-40.

25. Stan DL, Collins NM, Olsen MM, et al. The evolution of mindfulness-based physical interventions in breast cancer survivors. Evid Based Complement Alternat Med 
2012;2012:758641.

26. Carroll BJ, Iranmanesh A, Keenan DM, et al. Pathophysiology of hypercortisolism in depression: pituitary and adrenal responses to low glucocorticoid feedback. Acta Psychiatr Scand 2012;125:478-91.

27. Streeter CC, Gerbarg PL, Saper RB, et al. Effects of yoga on the autonomic nervous system, gamma-aminobutyricacid, and allostasis in epilepsy, depression, and posttraumatic stress disorder. Med Hypotheses 2012;78:571-9.

28. Rivest-Gadbois E, Boudrias MH. What are the known effects of yoga on the brain in relation to motor performances, body awareness and pain? A narrative review. Complement Ther Med 2019;44:129-42.

29. Amritanshu RR, Rao RM, Nagaratna R, et al. Effect of Long-term Yoga Practice on Psychological outcomes in Breast Cancer Survivors. Indian J Palliat Care 2017;23:231-6.

30. Rao RM, Vadiraja HS, Nagaratna R, et al. Effect of Yoga on Sleep Quality and Neuroendocrine Immune Response in Metastatic Breast Cancer Patients. Indian J Palliat Care 2017;23:253-60

31. Vadiraja HS, Rao RM, Nagarathna R, et al. Effects of Yoga in Managing Fatigue in Breast Cancer Patients: A Randomized Controlled Trial. Indian J Palliat Care 2017;23:247-52.

32. Danhauer SC, Griffin LP, Avis NE, et al. Feasibility of implementing a community-based randomized trial of yoga for women undergoing chemotherapy for breast cancer. J Community Support Oncol 2015;13:139-47.

33. Lanctôt D, Dupuis G, Marcaurell R, et al. The effects of the Bali Yoga Program (BYP-BC) on reducing psychological symptoms in breast cancer patients receiving chemotherapy: results of a randomized, partially blinded, controlled trial. J Complement Integr Med 2016;13:405-12.

34. Taso CJ, Lin HS, Lin WL, et al. The effect of yoga exercise on improving depression, anxiety, and fatigue in women with breast cancer: a randomized controlled trial. J Nurs Res 2014;22:155-64.

35. Jong MC, Boers I, Schouten van der Velden AP, et al. A Randomized Study of Yoga for Fatigue and Quality of Life in Women with Breast Cancer Undergoing (Neo) Adjuvant Chemotherapy. J Altern Complement Med 2018;24:942-53.

36. Jin CF, Wang LL, Wang B. The effect of yoga on cancer related fatigue and quality of life in breast cancer patients during chemotherapy. Nursing of Integrated Traditional Chinese and Western Medicine 2017;3:12-5.

37. Wang GF. The effect of yoga on cancer related fatigue and quality of life in breast cancer patients with chemotherapy [graduation thesis]: Central South University; 2013.

38. Chaoul A, Milbury K, Spelman A, et al. Randomized trial of Tibetan yoga in patients with breast cancer undergoing chemotherapy. Cancer 2018;124:36-45.

39. Cramer H, Lauche R, Klose P, et al. Yoga for improving health-related quality of life, mental health and cancerrelated symptoms in women diagnosed with breast cancer. Cochrane Database Syst Rev 2017;1:CD010802.

40. Higgins JP, Green S. Cochrane Handbook for Systematic Reviews of Interventions Version 5.1.0 [updated March 2011]. The Cochrane Collaboration 2011.

41. Moher D, Liberati A, Tetzlaff J, et al. Preferred reporting items for systematic reviews and meta-analyses: the PRISMA statement. BMJ 2009;339:b2535.

42. Higgins JP, Altman DG, Gøtzsche PC, et al. The Cochrane Collaboration's tool for assessing risk of bias in randomised trials. BMJ 2011;343:d5928.

43. Higgins JP, Thompson SG. Quantifying heterogeneity in a meta-analysis. Stat Med 2002;21:1539-58.

44. Lu G, Ades AE. Combination of direct and indirect evidence in mixed treatment comparisons. Stat Med 2004;23:3105-24.

45. Hozo SP, Djulbegovic B, Hozo I. Estimating the mean and variance from the median, range, and the size of a sample. BMC Med Res Methodol 2005;5:13.

46. Lu X, Yang H, Xia X, et al. Interactive Mobile Health Intervention and Blood Pressure Management in Adults. Hypertension 2019;74:697-704.

47. Feuerstein G. Chapter 1. The Yoga Tradition: Its History, Literature, Philosophy and Practice. Boston, MA: Hohm Press, 2001.

48. Garfinkel M, Schumacher HR Jr. Yoga. Rheum Dis Clin North Am 2000;26:125-32, x.

49. Feuerstein G. Chapter 1. The Deeper Dimension of Yoga: Theory and Practice. Boston, MA Prescott: Hohm Press, 2003.

50. Bock BC, Thind H, Fava JL, et al. Feasibility of yoga as a complementary therapy for patients with type 2 diabetes: The Healthy Active and in Control (HA1C) study. Complement Ther Med 2019;42:125-31.

51. Aljasir B, Bryson M, Al-Shehri B. Yoga Practice for the Management of Type II Diabetes Mellitus in Adults: A systematic review. Evid Based Complement Alternat Med 2010;7:399-408.

52. Raghavendra P, Shetty P, Shetty S, et al. Effect of highfrequency yoga breathing on pulmonary functions in patients with asthma: A randomized clinical trial. Ann 
Allergy Asthma Immunol 2016;117:550-1.

53. Bahçecio lu Turan G, Tan M. The effect of yoga on respiratory functions, symptom control and life quality of asthma patients: A randomized controlled study. Complement Ther Clin Pract 2020;38:101070.

54. Dunne EM, Balletto BL, Donahue ML, et al. The benefits of yoga for people living with HIV/AIDS: A systematic review and meta-analysis. Complement Ther Clin Pract 2019;34:157-64.

55. Hägglund E, Hagerman I, Dencker K, et al. Effects of yoga versus hydrotherapy training on health-related quality of life and exercise capacity in patients with heart failure: A randomized controlled study. Eur J Cardiovasc Nurs 2017;16:381-9.

56. Sharma S, Pailoor S, Choudhary Ram N, et al. Development of a yoga module targeting cardiovascular health for patients with post-myocardial left ventricular dysfunction in India. Complement Ther Med 2019;42:170-7.

57. Chanta A, Klaewsongkram J, Mickleborough TD, et al. Effect of Hatha yoga training on rhinitis symptoms and cytokines in allergic rhinitis patients. Asian Pac J Allergy Immunol 2019. [Epub ahead of print].

58. Chaturvedi A, Nayak G, Nayak AG, et al. Comparative Assessment of the Effects of Hatha Yoga and Physical Exercise on Biochemical Functions in Perimenopausal Women. J Clin Diagn Res 2016;10:KC01-4.

59. Kaur G, Prakash G, Malhotra P, et al. Home-Based Yoga Program for the Patients Suffering from Malignant Lymphoma during Chemotherapy: A Feasibility Study. Int J Yoga 2018;11:249-54.

60. Komatsu H, Yagasaki K, Yamauchi H, et al. A self-directed home yoga programme for women with breast cancer during chemotherapy: A feasibility study. Int J Nurs Pract 2016;22:258-66.

61. Kothari TO, Jakhar SL, Bothra D, et al. Prospective randomized trial of standard antiemetic therapy with yoga versus standard antiemetic therapy alone for highly emetogenic chemotherapy-induced nausea and vomiting in South Asian population. J Cancer Res Ther 2019;15:1120-3.

62. Chandwani KD, Thornton B, Perkins GH, et al. Yoga improves quality of life and benefit finding in women undergoing radiotherapy for breast cancer. J Soc Integr Oncol 2010;8:43-55.

63. Anampa J, Makower D, Sparano JA. Progress in adjuvant chemotherapy for breast cancer: an overview. BMC Med 2015;13:195.

64. Tao JJ, Visvanathan K, Wolff AC. Long term side effects of adjuvant chemotherapy in patients with early breast cancer. Breast 2015;24 Suppl 2:S149-53.

65. Lorusso D, Bria E, Costantini A, et al. Patients' perception of chemotherapy side effects: Expectations, doctor-patient communication and impact on quality of life - An Italian survey. Eur J Cancer Care (Engl) 2017;26. doi: 10.1111/ ecc. 12618 .

66. Mehnert A, Scherwath A, Schirmer L, et al. The association between neuropsychological impairment, selfperceived cognitive deficits, fatigue and health related quality of life in breast cancer survivors following standard adjuvant versus high-dose chemotherapy. Patient Educ Couns 2007;66:108-18.

67. van Waart H, Stuiver MM, van Harten WH, et al. Effect of Low-Intensity Physical Activity and Moderateto High-Intensity Physical Exercise During Adjuvant Chemotherapy on Physical Fitness, Fatigue, and Chemotherapy Completion Rates: Results of the PACES Randomized Clinical Trial. J Clin Oncol 2015;33:1918-27.

68. Courneya KS, Friedenreich CM, Quinney HA, et al. A longitudinal study of exercise barriers in colorectal cancer survivors participating in a randomized controlled trial. Ann Behav Med 2005;29:147-53.

69. Cramer H, Krucoff C, Dobos G. Adverse events associated with yoga: a systematic review of published case reports and case series. PLoS One 2013;8:e75515.

70. Cohen J. Statistical power analysis for the behavioral sciences. Academic Press; 2013.

71. Gothe NP, Keswani RK, McAuley E. Yoga practice improves executive function by attenuating stress levels. Biol Psychol 2016;121:109-16.

72. Banasik J, Williams H, Haberman M, et al. Effect of Iyengar yoga practice on fatigue and diurnal salivary cortisol concentration in breast cancer survivors. J Am Acad Nurse Pract 2011;23:135-42.

73. Vadiraja SH, Rao MR, Nagendra RH, et al. Effects of yoga on symptom management in breast cancer patients: A randomized controlled trial. Int J Yoga 2009;2:73-9.

74. Banerjee B, Vadiraj HS, Ram A, et al. Effects of an integrated yoga program in modulating psychological stress and radiation-induced genotoxic stress in breast cancer patients undergoing radiotherapy. Integr Cancer Ther 2007;6:242-50.

75. Esch T, Stefano GB, Fricchione GL, et al. The role of stress in neurodegenerative diseases and mental disorders. Neuro Endocrinol Lett 2002;23:199-208.

76. Vempati RP, Telles S. Yoga-based guided relaxation reduces sympathetic activity judged from baseline levels. 
Psychol Rep 2002;90:487-94.

77. Pullen PR, Nagamia SH, Mehta PK, et al. Effects of yoga on inflammation and exercise capacity in patients with chronic heart failure. J Card Fail 2008;14:407-13.

78. Schultz PE, Haberman M, Karatha K. Iyengar Yoga Can Promote Well-Being in Women Breast Cancer Survivors. Spokane, WA: Washington State University; 2007.

79. Liu L, Mills PJ, Rissling M, et al. Fatigue and sleep quality are associated with changes in inflammatory markers in breast cancer patients undergoing chemotherapy. Brain Behav Immun 2012;26:706-13.

80. Lyon DE, Cohen R, Chen H, et al. Relationship of systemic cytokine concentrations to cognitive function over two years in women with early stage breast cancer. J Neuroimmunol 2016;301:74-82.

81. van Vulpen JK, Schmidt ME, Velthuis MJ, et al. Effects of physical exercise on markers of inflammation in breast cancer patients during adjuvant chemotherapy. Breast Cancer Res Treat 2018;168:421-31.

82. Gopal A, Mondal S, Gandhi A, et al. Effect of integrated yoga practices on immune responses in examination stress - A preliminary study. Int J Yoga 2011;4:26-32.

83. Shete SU, Verma A, Kulkarni DD, et al. Effect of yoga training on inflammatory cytokines and C-reactive protein in employees of small-scale industries. J Educ Health Promot 2017;6:76.

84. Vijayaraghava A, Doreswamy V, Narasipur OS, et al. Effect of Yoga Practice on Levels of Inflammatory Markers After Moderate and Strenuous Exercise. J Clin Diagn Res 2015;9:CC08-12.

85. Berger BG, Owen DR. Mood alteration with yoga and swimming: aerobic exercise may not be necessary. Percept Mot Skills 1992;75:1331-43.

86. Carson JW, Carson KM, Porter LS, et al. Yoga for women with metastatic breast cancer: results from a pilot study. J Pain Symptom Manage 2007;33:331-41.

87. Cohen L, Warneke C, Fouladi RT, et al. Psychological adjustment and sleep quality in a randomized trial of the effects of a Tibetan yoga intervention in patients with lymphoma. Cancer 2004;100:2253-60.

88. Whisenant M, Wong B, Mitchell SA, et al. Symptom

Cite this article as: Yi LJ, Tian X, Jin YF, Luo MJ, JiménezHerrera MF. Effects of yoga on health-related quality, physical health and psychological health in women with breast cancer receiving chemotherapy: a systematic review and meta-analysis. Ann Palliat Med 2021;10(2):1961-1975. doi: 10.21037/apm-20-1484
Trajectories Are Associated With Co-occurring Symptoms During Chemotherapy for Breast Cancer. J Pain Symptom Manage 2019;57:183-9.

89. Mandelblatt JS, Zhai W, Ahn J, et al. Symptom burden among older breast cancer survivors: The Thinking and Living With Cancer (TLC) study. Cancer 2020;126:1183-92.

90. Schreier AM, Johnson LA, Vohra NA, et al. PostTreatment Symptoms of Pain, Anxiety, Sleep Disturbance, and Fatigue in Breast Cancer Survivors. Pain Manag Nurs 2019;20:146-51.

91. Ramachandran S, Sudhakar S, Thaslim F, et al. Beware! Reduced Functional Capacity and Quality of Life with Increased Fatigue Level among the Breast Cancer Survivors Undergoing Chemotherapy in India. Indian J Palliat Care 2018;24:117-8.

92. Iyengar BKS. Licht auf Yoga: Das grundlegende Lehrbuch des Hatha-Yoga. Germany, Hamburg: Nikol Verlagsges, 2014.

93. Cramer H, Lange S, Klose P, et al. Yoga for breast cancer patients and survivors: a systematic review and metaanalysis. BMC Cancer 2012;12:412.

94. Anestin AS, Dupuis G, Lanctot D, et al. The Effects of the Bali Yoga Program for Breast Cancer Patients on Chemotherapy-Induced Nausea and Vomiting: Results of a Partially Randomized and Blinded Controlled Trial. J Evid Based Complementary Altern Med 2017;22:721-30.

95. Lötzke D, Wiedemann F, Rodrigues Recchia D, et al. Iyengar-Yoga Compared to Exercise as a Therapeutic Intervention during (Neo)adjuvant Therapy in Women with Stage I-III Breast Cancer: Health-Related Quality of Life, Mindfulness, Spirituality, Life Satisfaction, and Cancer-Related Fatigue. Evid Based Complement Alternat Med 2016;2016:5931816.

96. Pan Y, Yang K, Wang Y, et al. Could yoga practice improve treatment-related side effects and quality of life for women with breast cancer? A systematic review and meta-analysis. Asia Pac J Clin Oncol 2017;13:e79-e95.

97. Rock CL, Doyle C, Demark-Wahnefried W. Nutrition and physical activity guidelines for cancer survivors. CA Cancer J Clin 2012;62:275-6. 